\title{
A comprehensive study to select suitable method to enhance mechanical properties during 3D printing of metallic materials
}

\author{
Utkarshika Chandra ${ }^{1}$, Rajesh Porwal $^{2}$ \\ ${ }^{1,2}$ Faculty of Mech Engineering, Institute of Tech SRMU, Lucknow 225003
}

\begin{abstract}
Metallic metals are governed by their mechanical properties. Ductility and Strength two most significant properties behave as two opposite poles while metal is manufactured by conventional methods. Additive manufacturing however acts as a bridge binding two into more of a proportional relationship. Additive manufacturing commonly referred as 3D Printing reduces the tact time along with high dimensional accuracies hence it has turned out to be the most researched technologies in the recent times. Out of several 3D Printing methods Selective laser melting and Electron beam melting prove more efficient in increasing the strength along with ductility by significantly altering the grain structure thus creating an array of properties which are different from their contemporaries. The paper deals with the effects of the two processes along with the challenges faced due to the defects risen during the processing in order to optimally decide the process to be taken up for a selected metallic metal
\end{abstract}

\section{Introduction}

DUCTILITY refers to the property possessed by a material where it has the ability to plastically deform before rupturing. It has been an important factor in determining the feasibility of a material when considered for its industrial use. The problem majorly faced is the inverse relationship between strength and ductility [1]. Hence a need for development of a method to eliminate this synergy is required, most preferable being $3 \mathrm{D}$ PRINTING. 3D PRINTING refers to the process of producing end product from CAD models by adding material layer by layer, often in a single step without the requirement of additional processing instead using rapid Prototyping [2]. Additive manufacturing or 3D printing process converts any model having a $3 \mathrm{D}$ definition into a real time exact version thus enabling a hassle free production environment where any shape and dimension can be manufactured without much alteration and resource investment.3D printing in its origin days was referred to as a process depositing binder material onto a powdered bed with inkjet heads layer by layer. Few of the different processes linked as follows:-

Fused deposition modelling this process involves adding Layers of melted plastic over one another. Materials mostly used are abs filament, polycarbonate, resin and nylon. Having a high complexity it runs on a fair speed along with fairly good accuracy that makes the material durable and ideal for making models with a sustainable surface finish. The only drawback of this process is that it has a low resolution. Still it finds its way for use in aviation industry for propellers or turbines etc. Selective laser sintering process involves arrangement of plastic powder layer by layer melted by the heat imparted by a laser [2]. Materials being used mainly are paper, plastic, glass, ceramics, composites etc. Having a lower complexity comparatively it's a fast and swift process with a good accuracy. It makes the material Resistant and durable. The only drawback it possesses is that the finished product needs post processing. It finds its way to automation industry. [4]. Stereo lithography process involves polymerization scanned by UV laser. Having used liquid photopolymer and composites as material, it has a medium level complex process. The speed being fast enough gives a very good dimensional accuracy as well as a very good surface finish. It can make complex geometries with a very high resolution. The only drawback is that it can only use photopolymer materials. Photopolymer jetting process uses inkjet method with liquid photopolymers. The raw material being metals, wax, plastic etc. having a moderate complexity the method is fast and applaud able for a good surface finish. The process shows an advantage of using many materials at the same time but the materials should be a photopolymer. The finished product however is not very durable. It finds its use in medico industry. Selective laser melting (SLM) involves using metal powder that is melted by laser. The materials involved are metals like copper aluminium etc. the complexity of the method is fairly low with an ok dimensional accuracy. And a satisfactory surface finish. It proves useful in manufacturing high 
density parts. The only drawback is the process being highly priced. It is used in manufacturing industrial components. [3]Electron beam melting (EBM) process involves using melted powder guided by an electron beam. Material used involves mostly metals with the process being fast and accurate but imparting an under satisfactory result in terms of surface finish. Still the method is preferred because it involves less thermal stress but can be used only on a set of metals. Mostly dental equipment's and medical implants are made using the process. [5]Electron binder jetting involves distributing powder with the help of jetting machine. It basically works with ceramics, metals, plastic, sand, composites etc. The process is fairly simple and swift but fragile with limited properties, giving a fair surface finish and dimensional accuracy to the end product. The end product requires no extra fittings and it gives multicolour prints. It is used in aerospace industry. Continuous fibre fabrication having a double nozzle melting method, uses plastic, nylon and composites. The process is however very complex with a fair finish and dimensional accuracy. It produces robust parts with no extra finishing required. Material jetting is an inkjet method with wax materials. The process is simple but slow in pace. The surface finish however is very good, with a good dimensional accuracy and a very high resolution. The only problem it possesses is that it can be only used in wax like materials it is used in making prototypes and castings e.g. lost wax casting.

\section{Literature review}

A Literature review is performed in order to gain a better insight of an existing topic. It helps to understand the topic from the perspective of an expert and get a hold of the ongoing research in the area. The analysis of various experts helps the reader to also go through the debates and contradictions which clears the air for a better understanding.

\subsection{Impact of process selection on ductility and strength of metallic materials}

Manufacturing processes are often broadly classified into conventional and non-conventional processes. When a conventional process of manufacturing for alloyed steel such as casting is used it is observed that there is a large presence of surface and corner micro cracking. For instance the crack presence and propagation that hinders the ductility of micro alloyed steel is mainly attributed to the presence of per eutectoid ferrite precipitated along the austenitic grain boundary. [6] The grains that are present are large and unevenly arranged with a high amount of boundary precipitation. In order to address this problem a process called DIRECT STRIP CASTING (DSC) was developed in Germany where inclusion of $\mathrm{Mn}, \mathrm{Si}$, and $\mathrm{Al}$ into the cast improved the tensile strength and ductility of Fe-Mn-Si-Al TWIP steels to 600-1200 MPa which was approx. 60-90\% more than rolling and annealing treatment but still this process could not be used by the general industries due to the limited knowledge of the properties of $\mathrm{Fe}-\mathrm{Mn}-\mathrm{Si}-\mathrm{Al} \mathrm{TWIP}$ and limited control of the casting process involved [7]. However the process highlighted the fact that high stacking fault energy which is the energy carried by stacking faults that basically interrupt the normal stacking of planes and dislocation planar guide with proper grain refinement and orientation can help tremendously in improving the strength and ductility of the metal.

\subsection{Role of 3d printing in increasing ductility and strength}

In $3 \mathrm{D}$ printing the most preferred process of additive manufacturing is SELECTIVE LASER MELTING. The complete fusion of the powder and the rapid cooling rate with a controlled thermal gradient makes the process viable for the usage. As discussed earlier the grain size and arrangement plays a significant role in improving both strength and ductility together. In conventional processing the introduction of crystalline structure is done to improve the strength but at the same time this crystalline structure alters the grain hierarchy leading to decrease in ductility. To address this Problem Speer developed a process wherein quenching and partitioning is used to improve strength and ductility of originally casted material. These steels mainly contain Fe ,C, Mn and $\mathrm{Si}$ which have relatively low cost, but in conclusion it was observed that the yield strength increased significantly while there was a moderate increment in ductility, also the process had various critical parameters to control which are yet to be researched upon[8].

It is known that a high temperature gradient with rapid heating and cooling is essential for required grain refinement. This thermal arc can be achieved in 3D printing. On analysis of $3 \mathrm{D}$ printing process when STAINLESS STEEL 316LSS is fabricated with the process of SELECTIVE LASER MELTING it was observed that there was a notable improvement in ductility and strength when compared with conventional processes. Wherein the tensile stress increased to anapprox. 551mpa as compared to $244 \mathrm{mpa}$ of wrought annealed 316LSS [9].The selective laser melting process has a distinctive characteristic of immediate cooling and heating while the mechanism happens. This results in an extremely fine granular structure of the particle as visible on studying a sample of $\mathrm{Al}-12 \mathrm{Si}$ where the microstructure consists of very fine $0.5-1 \mathrm{~mm}$ cellular aluminum surrounded by a network of nano-sized Si particles. This arrangement proves to be quite effective in increasing the ductility and strength.

\subsection{Impact of grain size on ductility and strength}

Grain growth in most of the materials conventionally formed follows the HALL PATCH effect which emphasizes on the phenomenon that the reduced grain size increases stress. The grain boundaries are incoherent thus it is difficult for one grain to force through another grain and move forward. This increase in strength comes at the cost of reduced ductility [9]. In SLM the structure of grains is epitaxial and columnar [10]. Which is due to 
high cooling rate thus SLM materials have a good homogeneity and refrain from formation of dendrites. On the other hand SLMed manufactured AlSi10Mg has a segregation of $\mathrm{Si}$ at the cell walls while CM247LC has $\mathrm{Hf} / \mathrm{Ti} / \mathrm{Ta} / \mathrm{W}$ segregation at the walls with the phase being eutectic in nature. This segregation is mainly due to solute redistribution which is greatly affected by solidification rate. Thus from studies it can be concluded that high solidification rate reduces the amount of solute redistribution and precipitation which in the case of steel is reduction in ferrite concentration which in turn increases the yield and tensile strength. The SLMed metals exhibit a columnar grain structure with a few dislocations embedded in them which are the solute precipitates as in the case of 316LSS [11]. When the cells expand the dislocation walls are formed with alloying elements being concentrated into the boundaries. These dislocations, mainly present as shock particles which often move in pairs increase the stacking fault dynamically [10]. These shock particles give a jerk to the motion of grains on application of external force, enough to hinder but not stop the motion. As the applied force increase the inter cell transfer of the particles thus increasing strength with no compromise in ductility. When the dislocations are absent the grains shear off one another which initiates cracks thus decreasing the UTS significantly. But apart from these dislocations there is another property called the TWINNING EFFECT. While the dislocations on the application of stress start gliding to each other, thereby assisting ductility they smash against the cells creating sufficient amount of shear stress too which helped in increasing the strength. As this is happening some dislocations get entangled with the cells thus lose their partners. Thus the left dislocations give rise to THE TWINNING EFFECT [8, 10, 11]. The TWINNING EFFECT is similar to the movement of a screw i.e. in a helical motion, gliding over one another thereby assisting the ductility. Hence it can be safely concluded that the ductility is a product of the gliding of the dislocations as well as twinning effect. The increased number of the dislocation also causes the presence of residual stress which imparts an internal strength to the structure, but in some cases for e.g.3161 Ti-6Al-4V, Nibased super alloys and Al-Si alloys these dislocations often cause a reduction in ductility and a brittle fracture. Too much presence of such dislocations can cause the localized straining hence early failure.

Along with the use of SLM the additive manufacturing process of ELECTRON BEAM MELTING is also used in some cases. The EBM manufactured TNM-B1 alloy displayed a strength of $462 \mathrm{MPa}$ and YS along with 580 MPa of UTS having ductility (58\% of El) at $800{ }^{\circ} \mathrm{C}$ [12]. These values are much higher than their conventionally manufactured counterparts. Just like SLM process the EBM manufactured parts displayed a columnar grain structure consisting of $\gamma, \alpha 2$ and $\beta$ o phases. Similarly in the case of EBM manufactured Alloy 718 when raster scanned along its hatch region also displayed columnar structure along the build direction [13]. The dramatic rise in ductility as well as strength in components manufactured by Additive manufacturing processes is due to presence of columnar, precipitate dislocated structure with the assistance of twinning effect.

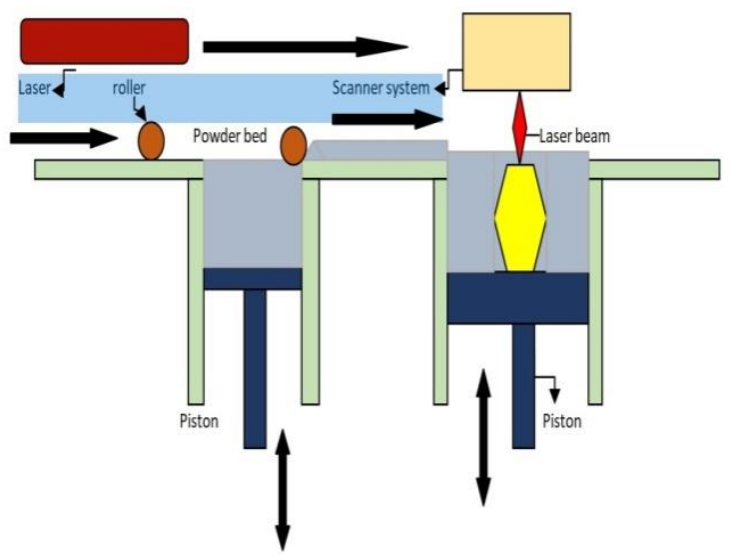

Fig 1: line diagram of a selective laser melting setup [6]

\subsection{Impact of porosity and residual stress on ductility and strength}

While considering the two processes i.e. SLM and EBM one cannot overlook the defects these processes inhibit in the structure. Scientists however focus on a major flaw that is very evident in SLM especially i.e. POROSITY. Porosity is the presence of pores in the contour space which can act as a crack nucleation as well as propagation source on application of external stress. The porosity is categorized mainly in three categories lack of fusion pores which are formed due to incomplete melting or fusion of particles and the remains of solid grains hinder the monitored columnar growth, gas bubbles which have trapped gas inside them either due to poor isolation of the heat affected zone from the environment or unstable melt flow and the third being key hole pores formed due to excessive energy concentration at a particular area [14, 15]. The porosity defect formed due to excessive laser power can be reduced by controlling the laser power at a point up till a certain critical value as it is observed that in metals like 3161 no effect on grain size was seen on reducing the energy density while a research at sun et al. showed considerable increase in ductility as well as strength on increasing laser power owing to a better refined grained structure. Another study at Montero Sisiga et al. showed complete u turn from previous study citing a weak texture and coarse grain structure with increase in power. Thus the effect and management laser power for different material according to their grain refinement is needed to be researcher upon further. When a study was conducted on $316 \mathrm{~L}$ stainless steel it caps concluded that low laser power considerably increased the UTS and the YS as well as the ductility. However it is said that low energy density causes formation of pores [16]. In this study however the pores so formed were smaller in size and could not act as crack initiation or nucleation sites and it was also observed that although an increase in energy density reduced pore formation but the number of pores formed stabilized after a critical value and remained almost constant from there. Thus the refined smaller grain 
structure dominated the system of stress propagation. This conclusion however cannot be generalized on all the elements as different element show different porosity structure according to their grain development. Hence more studies are required to get a clear insight on the matter.

Another defect that is considered to be dominant in SLMed structures is RESIDUAL STRESS. The rapid cooling taking place during the process is said to be the main reason behind the residual stress, however some amount of residual stress is regarded as desirable as it helps in giving internal strength to the material as explained above [17]. But proper cooling curve is advised to follow in order to reduce excess stress accumulation. Taking the case of EBM there is a complete decomposition of martensitic happening as well as the porosity is at a manageable level. If alloy 718 processes at El-Bagoury et al. is considered they applied a HIP as a post processing technique having a temperature range of $1100-1200 \mathrm{c}$ at $200 \mathrm{Mpa}$ for a time limit of 2 hours it was observed that the porosity levels reduced and the grain morphology also improved [13]. Post processing methods also include solution treatment method, machining hot Iso-static processing etc. Thus it can be said that in case of EBM processed elements post processing considerably helps.

The EBM process has a better record in residual stress than the SLM process where due to processing ion a controlled vacuum environment and elevated processing temperature the problem faced due to excess residual stresses is also reduced. The process taking place in a vacuum also reduces the chances of formation of oxide layer due to thermal decomposition over the surface.

One of the major issues faced during the study of the specimens was that most of the specimens examined were sliced out from the Hatch region. However most of the designs so formed in the real world are a definitive structure combining both hatch as well as contour. The conclusions made considered that the SLMed or the EBMed structure consists of columnar grains but in reality on doing a clearer introspection it's found that the contour region had a combination of a fine columnar grain which was elongated towards the direction of the contour as well as equiaxed grains. The shrinkage porosity however was less visible in the contour than the hatch region but the lack of fusion was considerably high in the contour region than the hatch region. Hence slicing the hatch region and coming on to conclusions while the contour has entirely different story could alter the conclusions completely hence it should be focused on in further studies to include all design considerations before drawing out conclusions $[18,19]$.

\section{Conclusion}

Any process thus developed has to come clean in all possible parameters when it comes to grading it as an optimal choice. But in real world just like Carnot's engine this is also a hypothesis as it is very difficult to control all the parameters of a process without affecting any other.
But the main aim of researchers is to create a path that goes through the middle. In this case that path is attained by creating a synergy between ductility and strength but keeping in mind other properties remain unaffected. Thus the following conclusions can be drawn upon and worked up on in order to deal with the shortcomings of the processes

1) In order to upgrade the performance of the product so formed unconventional methods prove to be a better option.

2) The complex equation between strength and ductility is simplified better by the use of additive manufacturing. Selective laser melting and electron beam melting prove to be an optimal choice when strength along with ductility are desired.

3) Slm and ebm have a columnar, equiexial structure with solute precipitation along the grain boundaries and a well functional Twinning structure which makes them desirable.

4)However in the case of selective laser melting the high cost and residual stress along with porosity make it less applicable universally

5) Electron beam melting due to the complete decomposition of the marten site phase the porosity does not become an issue with the end product.

6) The residual stress is not as significant and can be easily eliminated by post processing techniques such as quenching, annealing etc. but more research is required in order to find the optimal parametric conditions

7)Most of the experiments are worked upon the hatched wherein the hatched slice contains regularly arranged grains, the real disorder and defects lie in the contour region which has not columnar but curved columnar grains growing to the direction of the design. Hence in order to get a proper insight the contour region must also be studied for every design.

\section{References}

1. Lu\& K. The future of metals. Science, 328(5976), 319-320. (2010).

2. T.Duda, L.Raghavan, \& V. 3D metal printing technology. IFAC-Papers online, 49(29), 103-110 (2016).

3. C.Yap, C.Chua \& Z.Dong, An effective analytical model of selective laser melting, Virtual and Physical Prototyping, 11:1, 21-26, (2016)

4. A.Gratton, Comparison of mechanical, metallurgical properties of 17-4PH stainless steel between direct metal laser sintering (DMLS) and traditional manufacturing methods. (2012).

5. P.Narayana,C.Li,S.Kim,S.Kim,A.Marquardt, C. Leyens, N.Reddy, J. Yeom\&J.Hong,High strength and ductility of electron beam melted $\beta$ stabilized $\gamma$ TiAl alloy at $800^{\circ} \mathrm{C}$, Materials Science and Engineering: A,756,41-45(2019).

6. C. Du, J. Zhang, J. Wen, Y. Li \& P. Lan, Hot ductility trough elimination through single cycle of intense cooling and reheating for micro alloyed steel 
casting, Iron making\& Steelmaking,43:5,331$339,(2016)$

7. LAN, L. Song1, C. Du1, \& J. Zhang, Analysis of solidification microstructure and hot ductility of $\mathrm{Fe}-$ 22Mn-07C TWIP steelP.Materials Science and Technology, 30, (2014)

8. J. Fan, L.Zhu, J.Lu, T.Fu, A.hen, Theory of designing the gradient microstructured metals for overcoming strength-ductility trade-off,Scripta Materialia, 184,41-45, https://doi.org/10.1016/j.scriptamat.2020.03.045. (2020)

9. H.Wu\&G.Fan, An overview of tailoring strain delocalization for strength-ductility synergy, Progress in MaterialsScience(doi:https://doi.org/10.1016/j.pmat sci.2020.100675) (2020),

10. L.Liu, Q.Ding, Y.Zhong, JiZou, J.Wu, Y.Chiu, J. Li, Z.Zhang, Q.Yu\&Z.Shen,Dislocation network in additive manufactured steel breaks strengthductility trade-off, Materials Today, 21,354-361,( 2018)

11. Z.Zhu, Q.Nguyen ,F.Ng, X.An ,X.Liao, P.Liaw,S.Nai\&J.Wei Hierarchical Microstructure and strengthening mechanisms of a $\mathrm{CoCrFeNiMn}$ high entropy alloy additively manufactured by selective laser melting, Scripta Materialia,154,2024 (2018)

12. P.Narayana, C.Li, S.Kim, A.Marquardt, C. Leyens, N.Reddy, J.Yeom\&J.Hong, High strength and ductility of electron beam melted $\beta$ stabilized $\gamma$-TiAl alloy at $800^{\circ} \mathrm{C}$, Materials Science and Engineering: A,756,41-45,( 2019)

13. R.Arun,J.Moverare, S. Maha\&R.Pederson, Additive Manufacturing of Alloy 718 via Electron Beam Melting: Effect of Post-Treatment on the Microstructure and the Mechanical Properties. Materials,12(2018)

14. G.Wang,Q.Liu,H.Rao,H.Liu,C.Qiu.G.Wang,Q.Liu, H.Rao,H.Liu\&C.Qiu, Influence of porosity and microstructure on mechanical and corrosion properties of a selectively laser melted stainless steel,Journal of Alloys and Compounds, 831,154815 ,https://doi.org/10.1016/j.jallcom.2020.154815 (2020)

15. H.Bian, K.Aoyagi, Y.Zhao,C.Maeda,T.Mouri\& A. Chiba, Microstructure refinement for superior ductility of Al-Sialloy by electron beam melting, AdditiveManufacturing,Additive Manufacturing, 32(2020)

16. K.Prashanth, S.Scudino, and T.Maity, J.Das\& J. Eckert, Is the energy density a reliable parameter for materials synthesis by selective laser melting, Materials Research Letters(2017)

17. X.Jiang,W.Xiong, and L.Wang, M.Guo\&Z. Ding Heat treatment effects on microstructure-residual stress for selective laser melting $\mathrm{AlSi} 10 \mathrm{Mg}$, Materials Science and Technology, 36(2), 113(2019)

18. M.Cai, H.Huang, X.Zuo, H.Ding\& N Stanford ,Enhanced strength-ductility of medium Mn steel by quenching, partitioning and tempering, Materials
Science and Technology, DOI: 10.1080/02670836.2020.1719305 (2020):

19. P.Lan, \&L.Song, \&C.Du,\& J. Zhang, Analysis of solidification microstructure and hot ductility of $\mathrm{Fe}-$ 22Mn-0.7C TWIP steel. Materials Science andTechnology.30.1297-1304. (2014).

20. J. Pereira, E.Gil, L.Solaberrieta, M.Sebastián, Y. Bilbao \& P. Rodriguez,Comparison of AlSi7Mg0.6 alloy obtained by selective laser melting and investment casting processes: Microstructure and mechanical properties in as-built/as-cast and heattreated conditions, Materials Science and Engineering: A, 778,13912, (2020)

21. H.Yao, H.Tan, Z.He, D.Zhou, Z.Zhou, Z.Xue, Y., Yang \& Y. High strength and ductility AlCrFeNiV high entropy alloy with hierarchically heterogeneous microstructure prepared by selective laser melting. Journal of Alloys and Compounds, 813( 2020) 